\title{
Neuroprotective effect of sinapic acid on REV-ERB $\alpha$ modulated mitochondrial fission in Parkinson's disease
}

\author{
Sang-Bin LEE ${ }^{1}$ and Hyun Ok Yang ${ }^{1}$ \\ ${ }^{1}$ Korea Institute of Science and Technology
}

August 14, 2020

\begin{abstract}
Background and Purpose Parkinson's disease (PD) is the second most common neurodegenerative disease worldwide, and accumulating evidence indicates that mitochondrial dysfunction is associated with the progressive deterioration of this disease. Previous studies have shown that sinapic acid has a neuroprotective effect, but its mechanisms of action remain unclear. Experimental Approach The neuroprotective effect of sinapic acid was assayed in a PD mouse model generated by the neurotoxin 1-methyl-4-phenyl-1,2,3,6-tetrahydropyridine (MPTP), as well as in SH-SY5Y cells. Target protein expression was detected by western blotting and immunofluorescence. Key Results Sinapic acid treatment attenuated the behavioural defects and loss of dopaminergic neurons in the MPTP mouse model of PD. Sinapic acid also improved mitochondrial function in MPTP-treated mice, as indicated by diminished mitochondrial swelling and increased mitochondrial glutamate dehydrogenase activity. MPTP treatment increased the abundance of mitochondrial fission proteins such as dynamin-related protein 1 (Drp1) and phospho-Drp1 Ser616, while sinapic acid treatment attenuated abnormal mitochondrial fission. In addition, MPTP decreased the expression of REV-ERB $\alpha$ protein, whereas sinapic acid increased its expression. To elucidate the molecular mechanism linking REV-ERB $\alpha$ and mitochondrial fission, we used the pharmacological REV-ERB $\alpha$ inhibitor SR8278. Sinapic acid and SR8278 co-treatment reversed phospho-Drp1 Ser616 protein expression and the protective effect of sinapic acid in MPTP-treated mice. Conclusion and Implications Our findings demonstrated that sinapic acid protects against MPTP-induced PD by inhibiting abnormal mitochondrial fission through REV-ERB $\alpha$.
\end{abstract}

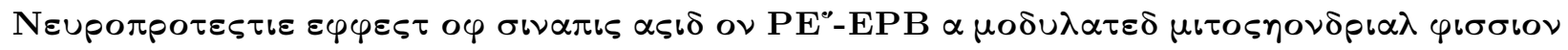

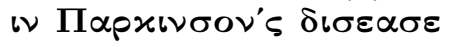

Short running title: Protective effect of sinapic acid on Parkinson's disease

Sang-Bin Lee ${ }^{1,2}$, Hyun Ok Yang ${ }^{1,3}$ *

${ }^{1}$ Natural Products Research Center, Korea Institute of Science and Technology, Gangneung 25451, Republic of Korea

2 School of Pharmacy, Sungkyunkwan University, Suwon 16419, Republic of Korea

${ }^{3}$ Division of Bio-Medical Science \& Technology, KIST School, Korea University of Science and Technology, Seoul 02792, Republic of Korea

* Corresponding author

Hyun Ok Yang, Ph.D.

Natural Products Research Center, Korea Institute of Science and Technology, Gangneung 25451, Korea

Tel: $+82-33-650-3501$

Fax: +82-33-650-3529 
E-mail: hoyang@kist.re.kr

Keywords: Parkinson's disease, Sinapic acid, Mitochondrial fission, REV-ERB $\alpha$

\section{Bullet point summary}

What is already known

Sinapic acid has been reported to have a neuroprotective effect in a 6-hydroxydopamine-induced animal model of parkinsonism.

What this study adds

Sinapic acid protected against MPTP-induced parkinsonism through REV-ERB $\alpha$-modulated mitochondrial fission.

Clinical significance

Sinapic acid could be a promising clinical candidate for the treatment of Parkinson's disease.

Abstract

Background and Purpose

Parkinson's disease (PD) is the second most common neurodegenerative disease worldwide, and accumulating evidence indicates that mitochondrial dysfunction is associated with the progressive deterioration of this disease. Previous studies have shown that sinapic acid has a neuroprotective effect, but its mechanisms of action remain unclear.

\section{Experimental Approach}

The neuroprotective effect of sinapic acid was assayed in a PD mouse model generated by the neurotoxin 1methyl-4-phenyl-1,2,3,6-tetrahydropyridine (MPTP), as well as in SH-SY5Y cells. Target protein expression was detected by western blotting and immunofluorescence.

\section{Key Results}

Sinapic acid treatment attenuated the behavioural defects and loss of dopaminergic neurons in the MPTP mouse model of PD. Sinapic acid also improved mitochondrial function in MPTP-treated mice, as indicated by diminished mitochondrial swelling and increased mitochondrial glutamate dehydrogenase activity. MPTP treatment increased the abundance of mitochondrial fission proteins such as dynamin-related protein 1 (Drp1) and phospho-Drp1 Ser616, while sinapic acid treatment attenuated abnormal mitochondrial fission. In addition, MPTP decreased the expression of REV-ERB $\alpha$ protein, whereas sinapic acid increased its expression. To elucidate the molecular mechanism linking REV-ERB $\alpha$ and mitochondrial fission, we used the pharmacological REV-ERB $\alpha$ inhibitor SR8278. Sinapic acid and SR8278 co-treatment reversed phosphoDrp1 Ser616 protein expression and the protective effect of sinapic acid in MPTP-treated mice.

\section{Conclusion and Implications}

Our findings demonstrated that sinapic acid protects against MPTP-induced PD by inhibiting abnormal mitochondrial fission through REV-ERB $\alpha$.

\section{Abbreviations}

PD, Parkinson's disease; Drp1, dynamic-related protein; MPTP, 1-methyl-4-phenyl-1,2,3,6tetrahydropyridine; SNpc, substantia nigra pars compacta

\section{Introduction}

Parkinson's disease (PD) is the second most common neurodegenerative disease and is characterized by progressive loss of dopaminergic neurons in the midbrain, especially the substantia nigra pars compacta (SNpc), with the formation of $\alpha$-synuclein aggregates called Lewy bodies (Duty \& Jenner, 2011). Mitochondrial 
dysfunction is considered one of the key pathogenic events of PD and contributes to the degeneration of dopaminergic neurons by activating inflammation and oxidative stress. Several mechanisms, especially mitochondrial fission and fusion, provide quality control through the recovery and/or elimination of damaged mitochondria, but an imbalance of these mechanisms could lead to the development of disease (Shirihai, Song \& Dorn, 2015). Thus, modulation of mitochondrial fission and fusion might be a promising candidate for therapeutic intervention in PD.

The term "circadian rhythms" refers to the physiologic, metabolic, and behavioural rhythms that follow a daily cycle. When circadian rhythms are disrupted, the regulation of sleep-wake cycles and immunity is disrupted as well, increasing susceptibility to sleep disturbances, infections and inflammatory disease (Carter et al., 2016). Sleep-wake disturbances are a very frequent and troublesome symptom in a number of PD patients (Ylikoski, Martikainen, Sieminski \& Partinen, 2015). The relation between circadian rhythms and mitochondria has been reported in various studies. Li et al. showed that Bmal1, the core circadian gene, controlled the development of dilated cardiomyopathy through regulation of mitochondrial fission and mitophagy (Li et al., 2020). Clock, which is another core circadian gene, modulates mitochondrial apoptosis by controlling mitochondrial membrane potential and permeabilization (Yang et al., 2020). Circadian rhythms are regulated by an autoregulatory transcriptional feedback loop including the proteins ROR $\alpha, \mathrm{ROR} \beta, \mathrm{REV}$ ERB $\alpha$ and REV-ERB $\beta$, which form a supporting loop to stabilize the main loop by regulating the expression of circadian rhythm proteins. The protein expression of Bmal1 is controlled by Rev response elements in a Baml1-Rev feedback loop (Ueda et al., 2005). Recently, components of the supporting loop have been recognized as important in the development of disease. SR9009, an agonist of REV-ERB $\alpha$, improved cardiac function through the regulation of inflammation and cardiac remodelling (Stujanna et al., 2017). In an animal model of Alzheimer's disease, SR9009 also decreased cognitive deficits and $\beta$-amyloid burden (Roby et al., 2019). Furthermore, REV-ERB $\alpha$ modulated mitochondrial biogenesis via the Stk11-AMPK-SIRT1PGC1 signalling pathway (Woldt et al., 2013b). Nevertheless, the relationship between REV-ERB $\alpha$ and mitochondrial fission is not fully understood.

Sinapic acid (SA) is a hydroxycinnamic acid-derived polyphenol with 3,5-dimethoxyl and 4-hydroxyl substitutions in the phenyl group of cinnamic acid. It is widely found in various plant-derived foods, such as fruits, vegetables, cereals and oilseed crops. Several studies have suggested that SA has anti-inflammatory, antioxidant, antimicrobial, and neuroprotective effects (Li et al., 2019; Shahmohamady, Eidi, Mortazavi, Panahi \& Minai-Tehrani, 2018). Kim et al. discovered that SA could attenuate kainic acid-induced hippocampal neuronal damage by suppressing reactive gliosis (Kim et al., 2010). In addition, SA also showed anxiolytic-like effects by acting on $\mathrm{GABA}_{\mathrm{A}}$ receptors and potentiating $\mathrm{Cl}^{-}$currents (Yoon et al., 2007). Recently, Zare et al. showed that SA inhibited the loss of dopaminergic neurons in a 6-OHDA-induced PD rat model by decreasing oxidative stress and lowering nigral iron levels (Zare, Eidi, Roghani \& Rohani, 2015). However, the mechanism of its neuroprotective effects against PD has never been investigated. In this study, we determined the possible underlying mechanism of SA in PD by using in vivo and in vitro methods.

\section{Methods}

\section{Cell culture and treatment}

SH-SY5Y cells (ATCC, Cat\# CRL-2266, RRID:CVCL_0019) were cultured at 37 in a humidified atmosphere of $5 \% \mathrm{CO}_{2}$ and $95 \%$ air with DMEM supplemented with $10 \%$ heat-inactivated FBS and $1 \%$ penicillin and streptomycin. The cells were incubated for 1 hour prior to $\mathrm{MPP}^{+}$treatment with Sinapic acid (SA), SR8278 (SR, REV-ERB $\alpha$ antagonist) and GSK4112 (GSK, REV-ERB $\alpha$ agonist) depending on the experimental design. The stock solutions of each reagent were diluted to the final concentrations in medium prior to use.

\section{Cell viability assay}

Cell viability was detected by using MTT assay kit (EZ-Cytox kit, Cat\# EZ-3000, DAEILLAM Co, Ltd, Seoul, Republic of Korea) according to the instruction of manufacturer. Briefly, SH-SY5Y cells were seeded at a density of $6 \times 10^{4}$ cells.well $^{-1}$ in 96-well culture plate and incubated with each reagent and EZ-Cytox solution for $1 \mathrm{~h}$ at 37 . The absorbance of culture plate was measured at $450 \mathrm{~nm}$ with a microplate reader 
(TECAN, Switzerland).

\section{Animals}

Male C57BL/6 mice (weight 25-28 g, 7 weeks old, Orient Bio Inc, Seongnam, Korea, MGI Cat\# 5656552, RRID:MGI:5656552) were kept a full automatically temperature and humidity controlled room (22 $\pm 3,50 \%$, $12 \mathrm{~h}$ light and dark cycle) with free access to food and water. Animal experiments were followed by the Korea Institute of Science and Technology Animal Care Committee guidelines and approved by the Korea Institute of Science and Technology Animal Care and Use Committee (KIST-2018-077). Furthermore, animal studies are reported in compliance with the ARRIVE guidelines (McGrath \& Lilley, 2015).

The mice were randomly divided into 5 groups in each animal experiments. The group for the first study was composed like a vehicle-treated control group (1, Control), SA $20 \mathrm{mg} \cdot \mathrm{kg}^{-1}$-treated control group (2, SA20), vehicle-treated MPTP group (3), SA $10 \mathrm{mg} \cdot \mathrm{kg}^{-1}$-treated MPTP group (4, SA10), and SA $20 \mathrm{mg} \cdot \mathrm{kg}^{-1}$-treated MPTP group (5, SA20). In the second study, the 5 groups are vehicle-treated control group (1, Control), vehicle-treated MPTP group (2), Ropinirole $5 \mathrm{mg} \cdot \mathrm{kg}^{-1}$-treated MPTP group (3, RO), SA $20 \mathrm{mg} \cdot \mathrm{kg}^{-1}$-treated MPTP group (4, SA), and both SA $20 \mathrm{mg} \cdot \mathrm{kg}^{-1}$ and SR $25 \mathrm{mg} \cdot \mathrm{kg}^{-1}$-treated MPTP group (5, SA+SR). Mice were injected $30 \mathrm{mg} \cdot \mathrm{kg}^{-1} \mathrm{MPTP}$ (i.p.) dissolved in PBS on five consecutive days with or without SA (p.o), SR and ropinirole (i.p.), which were treated 30 min before MPTP injection. SR was intraperitoneal administered at 30 min before SA treatment in the combination of SA and SR group. The treatment dose of SA was followed by previous neurological studies and all reagents were dissolved in PBS, but SR was dissolved in 5:5:90 DMSO cremophor ${ }^{-1}$ PBS $^{-1}$ vehicle followed by previous study of Welch et al. (Kim et al., 2010; Lee et al., 2012; Welch, Billon, Valfort, Burris \& Flaveny, 2017). After the behavioral tests, the mice were euthanized with $\mathrm{CO}_{2}$ inhalation and then SNpc tissues were collected and stored at -80 .

\section{Motor coordination measurements}

After seven days the last MPTP injection, mice were tested the behavior ability using rotarod test and pole test. Motor performance was evaluated using rotarod apparatus. The mice were trained for 3 min 3 times a day during 3 consecutive days. The rotarod speed was gradually increased from $2 \mathrm{rpm}$ to $16 \mathrm{rpm}$ for all training days. In the test day, mice were performed the behavior ability test in 3 trials with the same procedure on the last training day and the average of 3 trials per test was considered as the final score. For the pole test, mice were placed on the top of vertical pole $(55 \mathrm{~cm}$ height, $8 \mathrm{~mm}$ diameter $)$ before starting the measurement. The time until mice descended to the floor completely was recorded as the time to turn (T-turn).

\section{Preparation of samples}

For other biomarkers measurement, we made the lysates using the SH-SY5Y cells and mice brains. The cells were collected after treatment and lysed with RIPA buffer (Cell Signaling Technology Inc.) supplemented with protease inhibitor cocktail (Roche, Penzberg, Germany) according to the manufacturer's instructions. The half of mice brain tissues, especially SNpc tissues, were homogenized using the IKA RW 20 digital homogenizer (IKA, Staufen, Germany) with PRO-PREP (iNtRON Biotechnology Inc., Seongnam, Korea) and then centrifuged at 13,000 rpm for 30 min at 4 .

\section{Western blot analysis}

The protein concentrations of lysates from cells and mice brains were detected by the Bradford method and then separated on 8-15\% SDS-PAGE. After transferring the gels to polyvinylidene difluoride (PVDF, Millipore Corp., Billerica, MA, USA) membranes, the membranes were incubated with $5 \%$ skim milk or $3 \%$ BSA. Membranes were incubated with the primary antibody (1:1000) overnight, and there were followed by horseradish peroxidase (HRP)-conjugated secondary antibodies (1:5000) for $1 \mathrm{~h}$. Immunoreactive bands were detected with SuperSignal West Femto Maximum Sensitivity Substrate kit (Thermo Scientific, Pierce Biotechnology, Rockford, Illinois, USA) using the LAS-4000 mini system (Fujifilm, Japan). The intensities of the bands were normalized to the GAPDH intensity using Multi Gauge software (Fujifilm). All experimental procedures conformed with British Journal of Pharmacologyguideline (Alexander et al., 2018). 


\section{Mitochondrial glutamate dehydrogenase activity}

The glutamate dehydrogenase (GDH) activity was measured using a commercial assay kit (Cat\# K729, Biovision, CA, USA) according to the instruction of manufacturer. The lysates were mixed with reaction reagents and then the absorbance of each samples were detected at $450 \mathrm{~nm}$. For calculation of GDH activity, these mixtures were incubated for 30 min and measured again the absorbance.

\section{ATP concentration}

The concentration of ATP was detected by a commercial ATP assay kit (Cat\# K354, Biovision). After deproteinization of lysates using a Deproteinization Sample Preparation Kit (Cat\# K808, Biovision) to rapidly block ATP consumption, deproteinized lysates and reagents mixed and put in the 96-well plate. The plate was incubated for 30 min at room temperature and then the absorbance was measured at $570 \mathrm{~nm}$.

\section{Immunohistochemistry}

The SNpc tissues from the other half of brain were fixed with $4 \%$ paraformaldehyde and sectioned at $20 \mu \mathrm{m}$ using a freezing cryostat (Thermo Fisher, MA, USA). After 30 min reacting with $3 \%$ hydrogen peroxide to eliminate endogenous peroxidase, the slices were incubated with both mouse REV-ERB $\alpha$ antibody (1:100) and rabbit TH antibody (1:100) overnight. Alexa Fluor 594-conjugated goat anti-mouse IgG antibody (1:200, Cat\# A11032, RRID:AB_2534091) and Alexa Fluor 488-conjugated goat anti-rabbit IgG antibody (1:200, Cat\# A10008) were used for secondary antibody and Diamidino-2-phenylindole (Cat\# C6628, SigmaAldrich, St. Louis, MO, USA) was used to stain cell nuclei. Representative images were captured using a confocal microscopy (Leica, Solms, Germany). All experimental procedures conformed withBritish Journal of Pharmacology guideline (Alexander et al., 2018).

\section{Data and statistical analysis}

The data and statistical analysis complied with the recommendations on experimental design and analysis in pharmacology made by theBritish Journal of Pharmacology (Curtis et al., 2018). Sample sizes subjected to statistical analysis at least 5 animals per group $(\mathrm{n}=6)$, where $\mathrm{n}=$ number of independent values. Data were analyzed with Prism 7.0 software (GraphPad Software, Inc., San Diego, CA, USA, RRID:SCR_002798) using one-way ANOVA followed by the Bonferroni test for multiple comparisons. The results are expressed as the means \pm S.E.M.

\section{Materials}

Sinapic acid (SA, Cat\# D7927), MPTP (Cat\# M0896) and $\mathrm{MPP}^{+}$(Cat\# D048) were obtain from SigmaAldrich. SR8278 (SR, Cat\# 4463) and GSK4112 (GSK, Cat\# 3663) were purchased from Tocris Bioscience (Bristol, UK). DMEM and FBS were obtained from Hyclone (Logan, UT, USA). Trypsin-EDTA and mixture of penicillin and streptomycin were purchased at GIBCO-BRL (Grand Island, NY, USA). Rabbit antiTH (Cat\# 2792S, RRID:AB_2303165), REV-ERB $\alpha$ (Cat\# 13418S, RRID:AB_2630359), p-Drp1 ser616 (Cat\# 3455S, RRID:AB_2085352), p-Drp1 ser637 (Cat\# 4867S, RRID:AB_10622027) and GAPDH (Cat\# 2118S, RRID:AB_561053) were purchased from Cell Signaling Technology Inc. (Boston, MA, USA). Antirabbit (Cat\# 7074S, RRID:AB_2099233) and mouse (Cat\# 7076S, RRID:AB_330924) horseradish peroxidase (HRP) linked IgG antibodies also obtain from Cell Signaling Technology Inc. Mouse anti-Drp1 antibody (Cat\# sc-271583, RRID:AB_10659110) was purchased from Santa Cruz Biotechnology Inc. (Santa Cruz, CA, USA).

\section{Nomenclature of Targets and Ligands}

Key protein targets and ligands in this article are hyperlinked to corresponding entries inhttp://www.guidetopharmacology.org, the common portal for data from the IUPHAR/BPS Guide to PHARMACOLOGY (Harding et al., 2018), and are permanently archived in the Concise Guide to PHARMACOLOGY 2019/20 (Alexander et al., 2019).

\section{Results}


Effect of SA on N-methyl-4-phenylpyridinium (MPP $\left.{ }^{+}\right)$-induced cytotoxicity in human SHSY5Y neuroblastoma cells

First, we investigated the cytotoxicity of SA in SH-SY5Y cells using an MTT assay. SA did not show any toxicity to cells at a variety of concentrations (from 1 to $40 \mu \mathrm{M}$ ) (data not shown). To examine the effect of SA on $\mathrm{MPP}^{+}$-induced cytotoxicity, we treated cells were treated with SA up to a concentration of $40 \mu \mathrm{M}$ with or without $2 \mathrm{mM} \mathrm{MPP}{ }^{+}$. Figure $1 \mathrm{~A}$ shows that the cell survival rate was significantly decreased by $2 \mathrm{mM} \mathrm{MPP}{ }^{+}$. However, SA treatment dose-dependently protected the inhibition of $\mathrm{MPP}^{+}$on the survival rate of SH-SY5Y cells. To further investigate the protective effects of SA, we measured the level of tyrosine hydroxylase (TH), which is an indicator of the abundance of dopaminergic neurons. As shown in Figure 1B, the protein expression of $\mathrm{TH}$ was significantly inhibited to approximately $51 \%$ in the $\mathrm{MPP}^{+}$group relative to the control group. This decrease was attenuated by SA in a dose-dependent manner. cellular ATP levels and glutamate dehydrogenase (GDH) activity are usually detected for the measurement of mitochondrial function. GDH is located in the mitochondrial matrix, and leakage of GDH from mitochondria indicates disruption of mitochondrial membrane integrity. Several studies have shown that the loss of GDH activity is associated with mitochondrial function, as with a reduction in ATP levels (Holownia, Chwiecko \& Farbiszewski, 1994; Lee, Youn, Jang \& Yang, 2019). After $\mathrm{MPP}^{+}$treatment, the cellular ATP level was significantly decreased to approximately $36 \%$ relative to the control group, while the SA $40 \mu \mathrm{M}$ treatment group showed recovery of the cellular ATP level (Figure 1C). The median GDH activity in the $\mathrm{MPP}^{+}$group markedly increased to 1.7 times that of the control group, and $40 \mu \mathrm{M}$ SA attenuated this increase (Figure 1D). To investigate how $\mathrm{SA}$ showed a protective effect against $\mathrm{MPP}^{+}$-induced neurotoxicity, we measured the protein expression of REV-ERB $\alpha$, a circadian clock component, as a potent regulatory mechanism of mitochondrial fission. The protein expression of REV-ERB $\alpha$ was significantly decreased to $72 \%$ in the MPTP group, while SA attenuated REV-ERB $\alpha$ protein expression (Figure 2A). To determine whether the protective effect of SA against $\mathrm{MPP}^{+}$-induced neurotoxicity is dependent on REV-ERB $\alpha$, we used the REV-ERB $\alpha$ agonist and antagonist with SA. As shown in Figure 2B and C, both REV-ERB $\alpha$ antagonist treatment and SA treatment diminished the protein expression of TH and REV-ERB $\alpha$, but agonist treatment with SA elicited no change compared with SA only.

\section{Effect of SA on MPTP-induced neurotoxicity in C57BL/6 mice}

We also determined the effect of SA on the MPTP-induced PD mouse model. First, motor dysfunction was tested using the rotarod test and pole test. In the rotarod test, the latency to fall was markedly decreased in the MPTP group (51.7 $\pm 11.3 \mathrm{~s})$ compared with the control group $(123.8 \pm 14.3 \mathrm{~s})$. After 10 and $20 \mathrm{mg} / \mathrm{kg}$ SA treatment, the latency to fall was increased 2.2- and 2.3-fold compared with MPTP alone, respectively (Figure 3A). The MPTP treatment group also impaired performance (12.2 $\pm 1.4 \mathrm{~s})$ compared with the control group $(7.5 \pm 0.3 \mathrm{~s})$ in the pole test. However, 10 and $20 \mathrm{mg} / \mathrm{kg}$ SA treatment improved the performance to approximately 86 and $72 \%$, respectively (Figure 3B). Similar to an in vitro experiment, we detected the protein expression of TH. Several studies showed that TH levels were reduced in the SNpc of MPTP-treated mice, recapitulating the main neuropathological characteristic of PD (Hayashi et al., 2013; Laloux et al., 2008). In this study, the protein expression of TH in the SNpc was significantly reduced to $39 \%$ in MPTPtreated mice compared with that in the control group. SA at $20 \mathrm{mg} / \mathrm{kg}$ protected the loss of dopaminergic neurons (Figure 3E). In the mitochondrial function assay using ATP level and GDH activity, these two factors decreased to $83 \%$ and $55 \%$ in the MPTP group compared with the control group, respectively. SA at $20 \mathrm{mg} / \mathrm{kg}$ also improved these changes (Figure 3C and D). The protein expression of REV-ERB $\alpha$ was also similar to the in vitro experimental results. The protein expression of REV-ERB $\alpha$ was approximately $64 \%$ lower than that of the control group, while $20 \mathrm{mg} / \mathrm{kg} \mathrm{SA}$ attenuated this decrease. (Figure 3F). Consistent with the western blotting results, SA recovered both the protein expression of REV-ERB $\alpha$ and the loss of dopaminergic neurons, as shown by immunofluorescent labelling of REV-ERB $\alpha$ and TH (Figure 3G).

Effect of SA on the protein expression of mitochondrial fission in C57BL/6 mice with MPTPinduced neurotoxicity

Mitochondria easily maintain their homeostasis through several maintenance mechanisms, such as mitochon- 
drial fission. However, excessive and aberrant mitochondrial fission promotes mitochondrial fragmentation and dysfunction, which are associated with the pathology of a number of neurodegenerative diseases (Qi, Qvit, Su \& Mochly-Rosen, 2013). In this study, MPTP treatment tended to increase Drp1 protein expression, while SA treatment reversed this increase (Figure 4A). Interestingly, the activated form of Drp1, phosphoDrp1 Ser617, significantly increased 1.9-fold in MPTP-treated mice compared with that in the control group, but the inactivated form of Drp1, phospho-Drp1 Ser637, did not change (Figure 4B and C). This increase was attenuated by SA. Furthermore, the ratio of phospho-Drp1 Ser616/637 also increased in the MPTP group, while the $40 \mu \mathrm{M} \mathrm{SA}$ treatment group showed normalization of this ratio (Figure 4D).

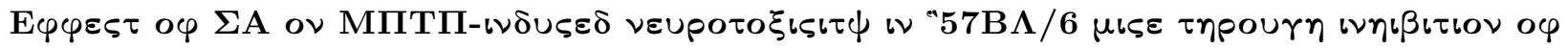 PE'-EPB $\alpha$}

Next, we investigated whether REV-ERB $\alpha$ was directly linked with the protective effect of SA in the MPTPinduced neurotoxicity mouse model. SR8278 (SR), a REV-ERB $\alpha$ antagonist, was employed to inhibit the protein expression of REV-ERB $\alpha$. In the motor function test, co-treatment with SA and SR markedly decreased motor performance compared with that of the SA treatment group in the rotarod test and pole test (Figure 5A and B). In addition, the protein expression of TH and REV-ERB $\alpha$ in the SNpc was markedly decreased to approximately 70 and $78 \%$ in the SA and SR co-treatment group compared with that in the SA group, respectively (Figure 5E and F). The ATP level and GDH activity also decreased to approximately $88 \%$ and $83 \%$, respectively, in the SA and SR co-treatment group compared with the SA group (Figure 5C and D). Consistent with the previous data, co-treatment with SA and SR showed an increasing tendency of Drp1 protein expression compared with the SA treatment group (Figure 6A). In addition, phospho-Drp1 Ser616 significantly increased 1.3-fold in both SA and SR-treated mice compared with the SA group, but phosphoDrp1 Ser637 did not change (Figure 6B and C). The ratio of phospho-Drp1 Ser616/637 also increased 1.2-fold in the SA and SR co-treatment group compared with the SA treatment group (Figure 6D).

\section{Discussion}

The protective effects of SA against neurodegenerative diseases and other diseases such as obesity and osteoarthritis have been studied, but there are no mechanistic studies regarding the effect of SA on circadian rhythms and mitochondrial function (Kim et al., 2010; Li et al., 2019; Yang et al., 2019; Zare, Eidi, Roghani \& Rohani, 2015). The present study demonstrates that SA ameliorates the neurotoxicity induced by MPTP and its metabolite $\mathrm{MPP}^{+}$through REV-ERB $\alpha$-modulated mitochondrial fission.

Mitochondrial dysfunction is closely linked to the pathogenesis of PD, and maintenance of mitochondrial function is considered a potential cure for the disease (Celardo, Martins \& Gandhi, 2014). MPTP is one of most commonly used chemical reagents for the PD model, and its treatment causes dopaminergic neuronal degeneration in the SNpc and behavioural abnormalities, which are based on mitochondrial damage. In the present study, MPTP or its metabolite $\mathrm{MPP}^{+}$-treated mice or cells increased dopaminergic neuronal damage by decreasing TH protein expression and mitochondrial damage by decreasing ATP levels and altering GDH activity. However, SA treatment restored TH protein expression and mitochondrial function. Additionally, SA also improved behavioural function in both tests using the MPTP-treated mouse model. Zare et al. reported that SA has neuroprotective potential against 6-OHDA in a PD animal model by regulating oxidative stress and lowering nigral iron levels (Zare, Eidi, Roghani \& Rohani, 2015). Our results confirmed that SA protects against dopaminergic neuronal cell damage and mitochondrial function.

Damaged mitochondria are regulated by several maintenance mechanisms, such as mitophagy, mitochondrial biogenesis, and mitochondrial fission and fusion, all working in coordination. Mitochondrial fission is considered the first step in the maintenance of mitochondrial homeostasis. However, excessive mitochondrial fission induces cell injury through ATP depletion, ROS generation and apoptosis activation, which are common causes of the development of neurodegenerative diseases (Roe \& Qi, 2018; Suen, Norris \& Youle, 2008). Drp1 is considered the most important protein in the fission process, and its activity is controlled by phosphorylation of serine 616 and serine 637 . An impaired balance between these two types of phosphorylation results in excessive mitochondrial fragmentation and neuronal cell death (Cho, Choi, Cho, Kim 
\& Sun, 2013). Recently, accumulating evidence has demonstrated that inhibition of Drp1 and/or phosphoDrp1 Ser616 ameliorates neurotoxicity and deficits in dopamine release in PD animal models. Simone et al. showed that treatment with mdivi-1, a putative inhibitor of Drp1, decreased mitochondrial dysfunction and oxidative stress in $\alpha$-synuclein-overexpressing rats (Bido, Soria, Fan, Bezard \& Tieu, 2017; Mishra, Singh, Tiwari, Bano \& Shukla, 2019; Park et al., 2019). Our findings demonstrate that MPTP-induced mitochondrial fission was significantly weaker after SA treatment through decreased protein expression of phospho-Drp1 Ser616 and is correlated with recovery of mitochondrial function. Therefore, our data suggested that SA attenuated excessive mitochondrial fission, especially the reduction of Drp1 phosphorylation at serine 616.

Circadian rhythms, which control the repeated approximately 24-hour sleep-wake cycle, manage various physiological functions, such as antioxidant and inflammatory responses, by changing gene expression (Zheng, Yuan, Wu, Lv \& Zhu, 2017). Thus, disruption of the circadian cycle is considered to be a key factor in several diseases, including cancer, metabolic disease, and neurodegenerative disease (Xie et al., 2019). The most well-known clinical characteristic of the abnormal circadian cycle is sleep disorder. For this reason, disruption of the circadian cycle is widely accepted as a novel risk factor for the development of PD because sleep and circadian disorders are easily discovered in PD patients (Videnovic \& Golombek, 2013). Several studies using animal PD models showed dopaminergic neuronal death and deregulated circadian symptoms, including behavioural and physiological outputs (Hayashi et al., 2013; Wang et al., 2018). Furthermore, MPTP treatment with environmental circadian disruption induced by long-term exposure to a 20:4 light/dark cycle exacerbated motor and cognitive deficits through excessive neuronal cell loss and the neuroinflammatory response (Lauretti, Di Meco, Merali \& Pratico, 2017). In addition, MPTP intoxication led to sleep disorders, including sleep episodes in the daytime and sleep fragmentation at night, whereas melatonin and L-dopa significantly improved these sleep symptoms in MPTP-treated monkeys (Belaid, Adrien, Karachi, Hirsch \& Francois, 2015). Collectively, these studies suggest that abnormal circadian and PD are closely related. REV-ERB $\alpha$ is responsible for the stability of the circadian rhythm by regulating circadian-related protein expression and is considered to be involved in the development of PD. Deletion of REV-ERB acontributed to exacerbated motor deficits and dopaminergic neuronal loss in a 6-OHDA-treated PD mouse model (Kim et al., 2018). In this study, SA treatment restored the protein level of REV-ERB $\alpha$ in MPTP-treated mice. Our data suggested that SA treatment has an effect on the recovery of circadian cycle disruption by regulating REV-ERB $\alpha$.

Although disruption of the circadian cycle and its contribution of onset to the development of PD were found in previous studies, whether disruption of the circadian cycle contributes to the progression of PD remains controversial. To adapt to the constant changes in the environment caused by daily changes during the day and night, a continuous supply of energy is essential to maintain and improve cell function (de Goede, Wefers, Brombacher, Schrauwen \& Kalsbeek, 2018). Thus, the regulation of mitochondrial function is considered a possible candidate for this argument. Interestingly, several studies have suggested that mitochondrial functions and morphologic changes are dependent on a viable circadian clock. Bmal1 knockout mice showed mitochondrial defects accompanying morphological changes and functional abnormalities, which led to severe, progressive, age-dependent heart failure (Kohsaka et al., 2014). Altered mitochondrial gene expression and increased mitochondrial oxidative stress were discovered in aged mice with mutations in the clock gene (Gong et al., 2015). Moreover, Woldt et al. demonstrated that genetic deletion of $\rho \epsilon-\epsilon \rho \beta$ a in mice resulted in impaired mitochondrial content and oxidative function; however, the amount of mitochondria and respiratory capacity were improved after treatment with the REV-ERB $\alpha$-expressing vector (Woldt et al., 2013a). To investigate whether the protective effect of SA is mediated by mitochondrial fission through the regulation of REV-ERB $\alpha$, we tested an antagonist and agonist of REV-ERB $\alpha$ with SA in an in vitro and in vivo PD model. The protective effect of SA disappeared after co-treatment with SA and REV-ERB $\alpha$ antagonists, as shown by decreased mitochondrial function and $\mathrm{TH}$ protein expression, while SA and agonist co-treatment did not change in the in vitromodel. These in vivo results using an antagonist with SA indicated that the recovery effect of SA was diminished, as shown by decreased behavioural function and TH protein expression. In addition, mitochondrial fission boosted after co-treatment with SA and antagonist. Our data also showed that the level of Drp1 protein expression driven by the plasmid was decreased after treatment with REV-ERB 
$\alpha$ recombinant protein (Figure S1). Together, these data support a prominent role for SA in the regulation of mitochondrial fission via the REV-ERB $\alpha$ protein.

In this study, we identified the pharmacological activation of SA in a neurotoxin-induced PD model through the regulation of REV-ERB $\alpha$ protein expression and a novel role of REV-ERB $\alpha$ in managing mitochondrial homeostasis by maintaining the balance of two kinds of Drp1 phosphorylation. Comprehensively, SA could be a promising therapeutic option for the prevention and treatment of PD.

\section{Acknowledgements}

This research was supported by a grant of the Korea Health Technology R\&D Project through the Korea Health Industry Development Institute (KHIDI), funded by the Ministry of Health \& Welfare, Republic of Korea (grant number: HI18C1860). This research was also supported by research funds from the National Research Foundation of Korea (NRF-2015-0001276) through the NRF funded by the Ministry of Education, Science, and Technology, Republic of Korea.

\section{Conflicts of interest}

The authors declare no competing financial interest.

\section{References}

Alexander SPH, Mathie A, Peters JA, Veale EL, Striessnig J, Kelly E, et al. (2019). THE CONCISE GUIDE TO PHARMACOLOGY 2019/20: Ion channels. Br J Pharmacol 176 Suppl 1: S142-S228.

Alexander SPH, Roberts RE, Broughton BRS, Sobey CG, George CH, Stanford SC, et al. (2018). Goals and practicalities of immunoblotting and immunohistochemistry: A guide for submission to the British Journal of Pharmacology. Br J Pharmacol 175: 407-411.

Belaid H, Adrien J, Karachi C, Hirsch EC, \& Francois C (2015). Effect of melatonin on sleep disorders in a monkey model of Parkinson's disease. Sleep Med 16: 1245-1251.

Bido S, Soria FN, Fan RZ, Bezard E, \& Tieu K (2017). Mitochondrial division inhibitor-1 is neuroprotective in the A53T-alpha-synuclein rat model of Parkinson's disease. Sci Rep 7: 7495.

Carter SJ, Durrington HJ, Gibbs JE, Blaikley J, Loudon AS, Ray DW, et al. (2016). A matter of time: study of circadian clocks and their role in inflammation. J Leukoc Biol 99: 549-560.

Celardo I, Martins LM, \& Gandhi S (2014). Unravelling mitochondrial pathways to Parkinson's disease. Br J Pharmacol 171: 1943-1957.

Cho B, Choi SY, Cho HM, Kim HJ, \& Sun W (2013). Physiological and pathological significance of dynaminrelated protein 1 (drp1)-dependent mitochondrial fission in the nervous system. Exp Neurobiol 22:149-157.

Curtis MJ, Alexander S, Cirino G, Docherty JR, George CH, Giembycz MA, et al. (2018). Experimental design and analysis and their reporting II: updated and simplified guidance for authors and peer reviewers. Br J Pharmacol 175: 987-993.

de Goede P, Wefers J, Brombacher EC, Schrauwen P, \& Kalsbeek A (2018). Circadian rhythms in mitochondrial respiration. J Mol Endocrinol 60: R115-r130.

Duty S, \& Jenner P (2011). Animal models of Parkinson's disease: a source of novel treatments and clues to the cause of the disease. Br J Pharmacol 164: 1357-1391.

Gong C, Li C, Qi X, Song Z, Wu J, Hughes ME, et al. (2015). The daily rhythms of mitochondrial gene expression and oxidative stress regulation are altered by aging in the mouse liver. Chronobiol Int 32: $1254-1263$. 
Harding SD, Sharman JL, Faccenda E, Southan C, Pawson AJ, Ireland S, et al. (2018). The IUPHAR/BPS Guide to PHARMACOLOGY in 2018: updates and expansion to encompass the new guide to IMMUNOPHARMACOLOGY. Nucleic Acids Res 46: D1091-d1106.

Hayashi A, Matsunaga N, Okazaki H, Kakimoto K, Kimura Y, Azuma H, et al. (2013). A disruption mechanism of the molecular clock in a MPTP mouse model of Parkinson's disease. Neuromolecular Med $15: 238-251$.

Holownia A, Chwiecko M, \& Farbiszewski R (1994). Accumulation of ammonia and changes in the activity of some ammonia metabolizing enzymes during brain ischemia/reperfusion injury in rats. Mater Med Pol 26: $25-27$.

Kim DH, Yoon BH, Jung WY, Kim JM, Park SJ, Park DH, et al.(2010). Sinapic acid attenuates kainic acid-induced hippocampal neuronal damage in mice. Neuropharmacology 59: 20-30.

Kim J, Jang S, Choi M, Chung S, Choe Y, Choe HK, et al. (2018). Abrogation of the Circadian Nuclear Receptor REV-ERBalpha Exacerbates 6-Hydroxydopamine-Induced Dopaminergic Neurodegeneration. Mol Cells 41: 742-752.

Kohsaka A, Das P, Hashimoto I, Nakao T, Deguchi Y, Gouraud SS, et al. (2014). The circadian clock maintains cardiac function by regulating mitochondrial metabolism in mice. PLoS One 9:e112811.

Laloux C, Derambure P, Houdayer E, Jacquesson JM, Bordet R, Destee A, et al. (2008). Effect of dopaminergic substances on sleep/wakefulness in saline- and MPTP-treated mice. J Sleep Res 17: 101-110.

Lauretti E, Di Meco A, Merali S, \& Pratico D (2017). Circadian rhythm dysfunction: a novel environmental risk factor for Parkinson's disease. Mol Psychiatry 22: 280-286.

Lee HE, Kim DH, Park SJ, Kim JM, Lee YW, Jung JM, et al. (2012). Neuroprotective effect of sinapic acid in a mouse model of amyloid beta(1-42) protein-induced Alzheimer's disease. Pharmacol Biochem Behav 103: 260-266.

Lee SB, Youn J, Jang W, \& Yang HO (2019). Neuroprotective effect of anodal transcranial direct current stimulation on 1-methyl-4-phenyl-1,2,3,6-tetrahydropyridine (MPTP)-induced neurotoxicity in mice through modulating mitochondrial dynamics. Neurochem Int 129: 104491.

Li E, Li X, Huang J, Xu C, Liang Q, Ren K, et al. (2020). BMAL1 regulates mitochondrial fission and mitophagy through mitochondrial protein BNIP3 and is critical in the development of dilated cardiomyopathy. Protein Cell.

Li X, Lin J, Ding X, Xuan J, Hu Z, Wu D, et al. (2019). The protective effect of sinapic acid in osteoarthritis: In vitro and in vivo studies. J Cell Mol Med 23: 1940-1950.

McGrath JC, \& Lilley E (2015). Implementing guidelines on reporting research using animals (ARRIVE etc.): new requirements for publication in BJP. Br J Pharmacol 172: 3189-3193.

Mishra A, Singh S, Tiwari V, Bano S, \& Shukla S (2019). Dopamine D1 receptor agonism induces dynamin related protein-1 inhibition to improve mitochondrial biogenesis and dopaminergic neurogenesis in rat model of Parkinson's disease. Behav Brain Res 378: 112304.

Park J, Seo J, Won J, Yeo HG, Ahn YJ, Kim K, et al. (2019). Abnormal Mitochondria in a Non-human Primate Model of MPTP-induced Parkinson's Disease: Drp1 and CDK5/p25 Signaling. Exp Neurobiol 28: 414-424.

Qi X, Qvit N, Su YC, \& Mochly-Rosen D (2013). A novel Drp1 inhibitor diminishes aberrant mitochondrial fission and neurotoxicity. J Cell Sci 126: 789-802.

Roby DA, Ruiz F, Kermath BA, Voorhees JR, Niehoff M, Zhang J, et al. (2019). Pharmacological activation of the nuclear receptor REV-ERB reverses cognitive deficits and reduces amyloid- $\beta$ burden in a mouse model 
of Alzheimer's disease. PLoS One 14: e0215004.

Roe AJ, \& Qi X (2018). Drp1 phosphorylation by MAPK1 causes mitochondrial dysfunction in cell culture model of Huntington's disease. Biochem Biophys Res Commun 496: 706-711.

Shahmohamady P, Eidi A, Mortazavi P, Panahi N, \& Minai-Tehrani D (2018). Effect of sinapic acid on memory deficits and neuronal degeneration induced by intracerebroventricular administration of streptozotocin in rats. Pol J Pathol 69: 266-277.

Shirihai OS, Song M, \& Dorn GW, 2nd (2015). How mitochondrial dynamism orchestrates mitophagy. Circ Res 116: 1835-1849.

Stujanna EN, Murakoshi N, Tajiri K, Xu D, Kimura T, Qin R, et al.(2017). Rev-erb agonist improves adverse cardiac remodeling and survival in myocardial infarction through an anti-inflammatory mechanism. PLoS One 12: e0189330-e0189330.

Suen DF, Norris KL, \& Youle RJ (2008). Mitochondrial dynamics and apoptosis. Genes Dev 22: 1577-1590.

Ueda HR, Hayashi S, Chen W, Sano M, Machida M, Shigeyoshi Y, et al. (2005). System-level identification of transcriptional circuits underlying mammalian circadian clocks. Nat Genet 37: 187-192.

Videnovic A, \& Golombek D (2013). Circadian and sleep disorders in Parkinson's disease. Exp Neurol 243: 45-56.

Wang Y, Lv D, Liu W, Li S, Chen J, Shen Y, et al. (2018). Disruption of the Circadian Clock Alters Antioxidative Defense via the SIRT1-BMAL1 Pathway in 6-OHDA-Induced Models of Parkinson's Disease. Oxid Med Cell Longev 2018: 4854732.

Welch RD, Billon C, Valfort AC, Burris TP, \& Flaveny CA (2017). Pharmacological inhibition of REV-ERB stimulates differentiation, inhibits turnover and reduces fibrosis in dystrophic muscle. Sci Rep 7: 17142.

Woldt E, Sebti Y, Solt LA, Duhem C, Lancel S, Eeckhoute J, et al.(2013a). Rev-erb-alpha modulates skeletal muscle oxidative capacity by regulating mitochondrial biogenesis and autophagy. Nat Med 19:1039-1046.

Woldt E, Sebti Y, Solt LA, Duhem C, Lancel S, Eeckhoute J, et al.(2013b). Rev-erb- $\alpha$ modulates skeletal muscle oxidative capacity by regulating mitochondrial biogenesis and autophagy. Nat Med 19:1039-1046.

Xie Y, Tang Q, Chen G, Xie M, Yu S, Zhao J, et al. (2019). New Insights Into the Circadian Rhythm and Its Related Diseases. Front Physiol 10: 682.

Yang C, Deng Q, Xu J, Wang X, Hu C, Tang H, et al. (2019). Sinapic acid and resveratrol alleviate oxidative stress with modulation of gut microbiota in high-fat diet-fed rats. Food Res Int 116:1202-1211.

Yang S, Liu Y, Guo Y, Liu R, Qi F, Li X, et al. (2020). Circadian gene Clock participates in mitochondrial apoptosis pathways by regulating mitochondrial membrane potential, mitochondria out membrane permeablization and apoptosis factors in AML12 hepatocytes. Mol Cell Biochem 467: 65-75.

Ylikoski A, Martikainen K, Sieminski M, \& Partinen M (2015). Parkinson's disease and insomnia. Neurol Sci 36: 2003-2010.

Yoon BH, Jung JW, Lee JJ, Cho YW, Jang CG, Jin C, et al. (2007). Anxiolytic-like effects of sinapic acid in mice. Life Sci 81:234-240.

Zare K, Eidi A, Roghani M, \& Rohani AH (2015). The neuroprotective potential of sinapic acid in the 6-hydroxydopamine-induced hemi-parkinsonian rat. Metab Brain Dis 30: 205-213.

Zheng JL, Yuan SS, Wu CW, Lv ZM, \& Zhu AY (2017). Circadian time-dependent antioxidant and inflammatory responses to acute cadmium exposure in the brain of zebrafish. Aquat Toxicol 182: 113-119.

Figure 1. Effect of SA in MPP ${ }^{+}$SH-SY5Y cells. (A) Evaluation of the dose-dependent effects of SA. (B) The protein expression of $\mathrm{TH}$ was measured by western blot after treatment with both $\mathrm{MPP}^{+}$and SA. (C) 
The cellular ATP content and (D) median GDH activity were measured using a commercially available assay kit to examine mitochondrial function. The results are presented as the mean \pm S.E.M. ${ }^{*} P<0.05$ compared to the control group, ${ }^{\#} p<0.05$ compared to the $\mathrm{MPP}^{+}$group.

Figure 2 . Effect of SA in $\mathrm{MPP}^{+}$-treated SH-SY5Y cells exposed to the REV-ERB $\alpha$ antagonist SR8278 and the REV-ERB $\alpha$ agonist GSK4112. (A - C) The protein expression of REV-ERB $\alpha$ and TH was measured by western blot after treatment with both $\mathrm{MPP}^{+}$and SA. The results are presented as the mean \pm S.E.M. ${ }^{*} P<0.05$ compared to the control group, ${ }^{\#} p<0.05$ compared to the $\mathrm{MPP}^{+}$group.

Figure 3. Effect of SA in MPTP-treated mice. (A - B) After 5 days of SA treatment with or without MPTP, we examined the motor function of mice using the rotarod test and the pole test. (C - D) The cellular ATP content and GDH activity were measured using a commercially available assay kit to examine mitochondrial function. (E - F) The protein expression of TH and REV-ERB $\alpha$ was measured by western blot after treatment with both MPTP and SA. (G) Images of TH (green), REV-ERB $\alpha$ (red) and nuclei (blue) in the SNpc tissues were collected by fluorescence microscopy. The results are presented as the mean \pm S.E.M. ${ }^{*} P<0.05$ compared to the control group, ${ }^{\#} p<0.05$ compared to the MPTP group.

Figure 4 Effect of SA on mitochondrial fission in MPTP-treated mice. (A - D) The protein expression of Drp1, phospho-Drp1 Ser616, and phospho-Drp1 Ser637 was measured by western blot after treatment with both MPTP and SA. The results are presented as the mean \pm S.E.M. ${ }^{*} P<0.05$ compared to the control group, ${ }^{\#} p<0.05$ compared to the MPTP group.

Figure 5 . Effect of SA in MPTP-treated mice through inhibition of REV-ERB $\alpha$.

$(\mathrm{A}-\mathrm{B})$ After 5 days of SA and SR treatment with or without MPTP, we examined the motor function of mice using the rotarod test and the pole test. (C - D) The cellular ATP content and GDH activity were measured using a commercially available assay kit to examine mitochondrial function. (E - F) The protein expression of TH and REV-ERB $\alpha$ was measured by western blot after treatment with MPTP, SA and SR. The results are presented as the mean \pm S.E.M. ${ }^{*} P<0.05$ compared to the control group, ${ }^{\#} p<0.05$ compared to the MPTP group, ${ }^{\$} p<0.05$ compared to the SA group.

Figure 6 . Effect of SA on mitochondrial fission in MPTP-treated mice through inhibition of REV-ERB $\alpha$. (A - D) The protein expression of Drp1, phospho-Drp1 Ser616, and phospho-Drp1 Ser637 was measured by western blot after treatment with MPTP, SA and SR. The results are presented as the mean \pm S.E.M. ${ }^{*} P<$ 0.05 compared to the control group, ${ }^{\#} p<0.05$ compared to the MPTP group, ${ }^{\$} p<0.05$ compared to the SA group. 
A

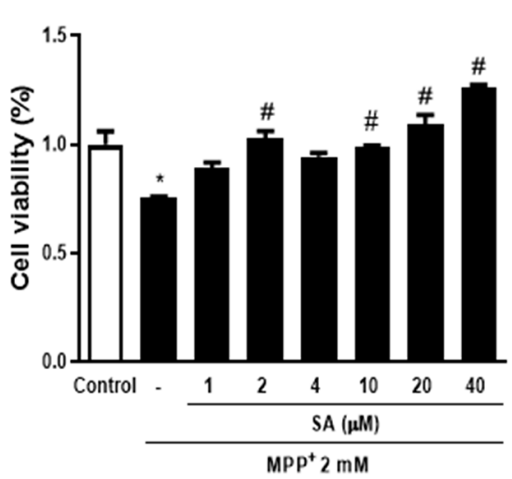

C

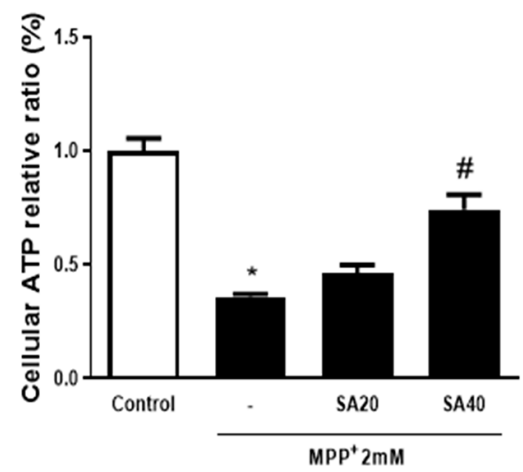

B

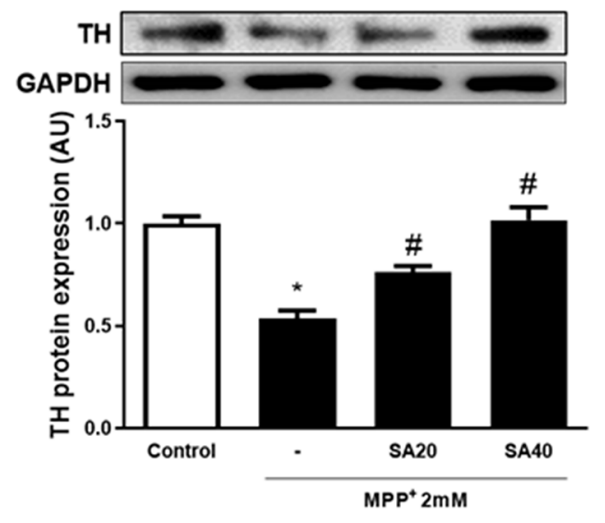

D

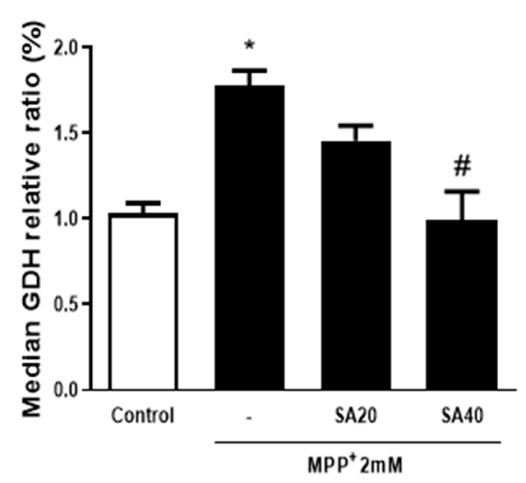


A
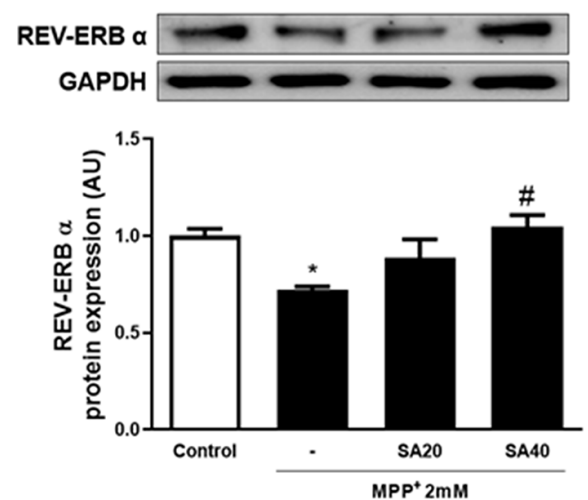

C

REV-ERB

GAPDH

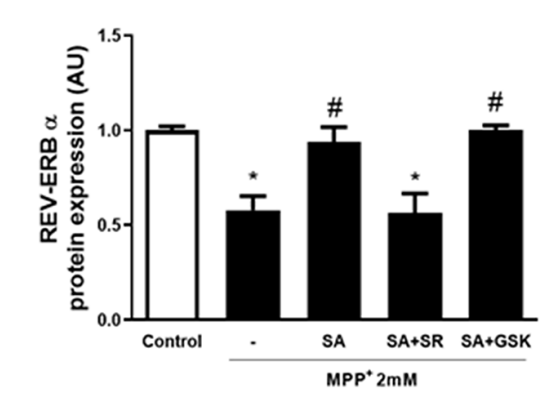

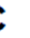

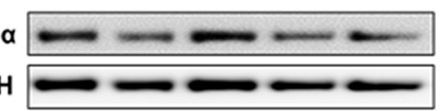

B
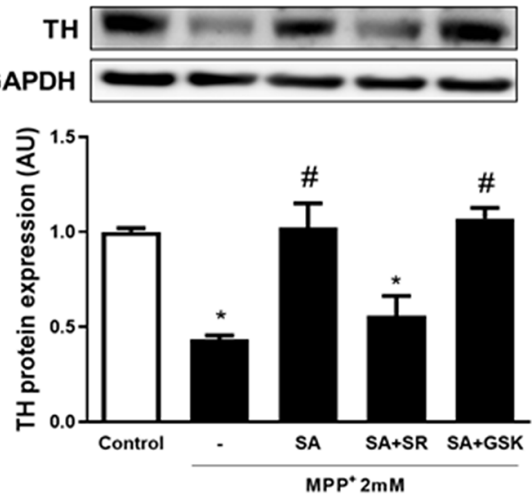
A

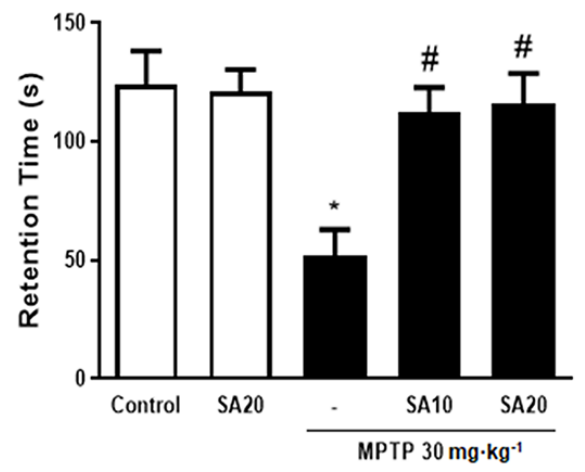

C
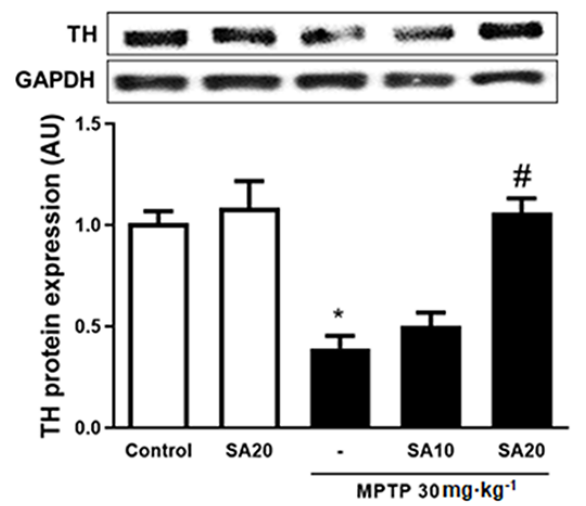

E

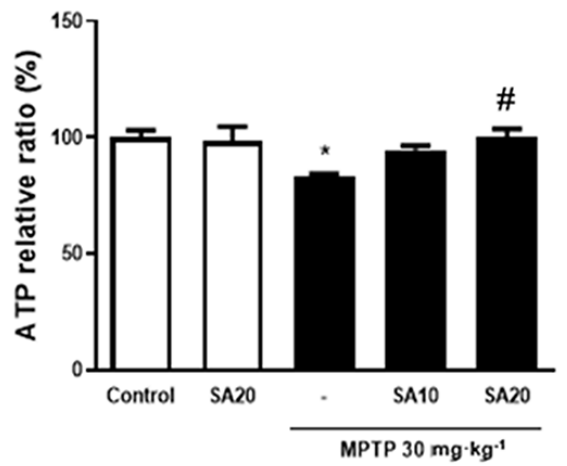

B

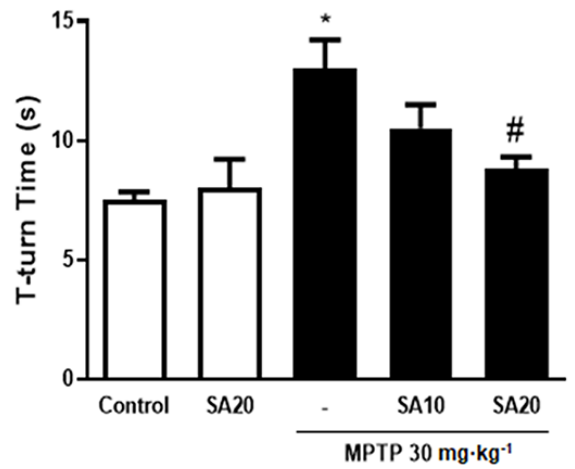

D

REV-ERB GAPDH

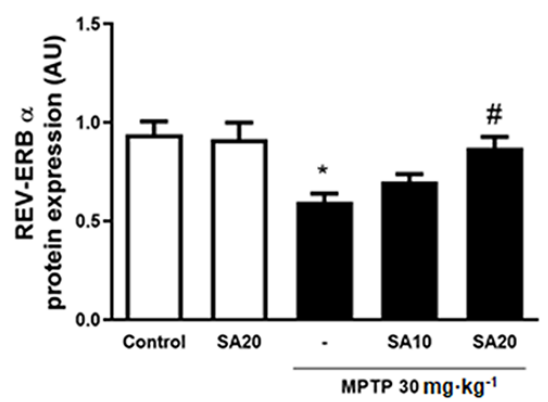

F

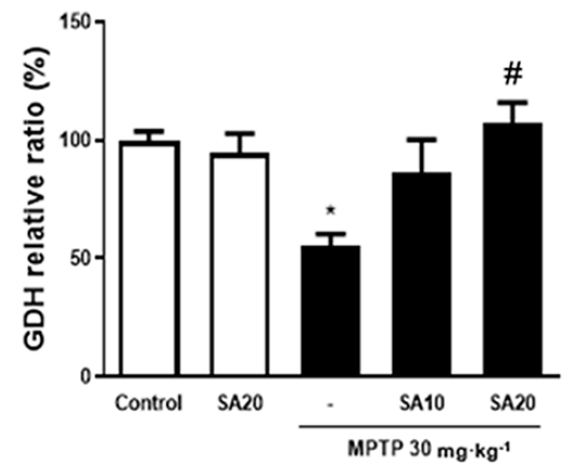

G

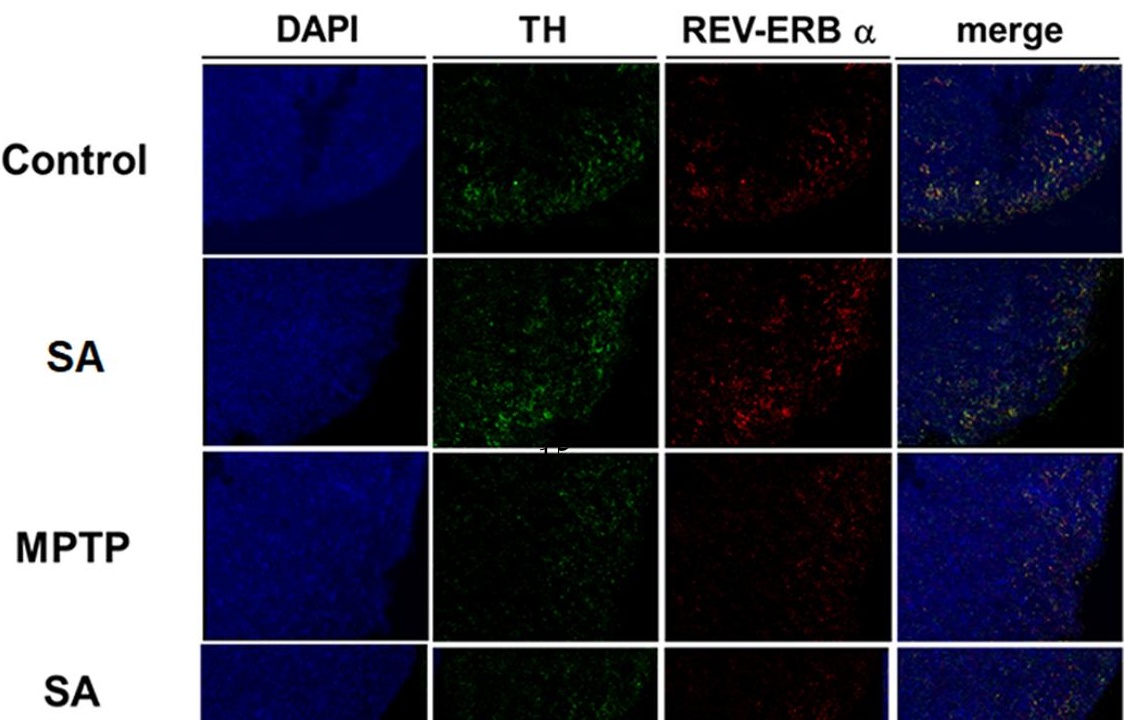


A
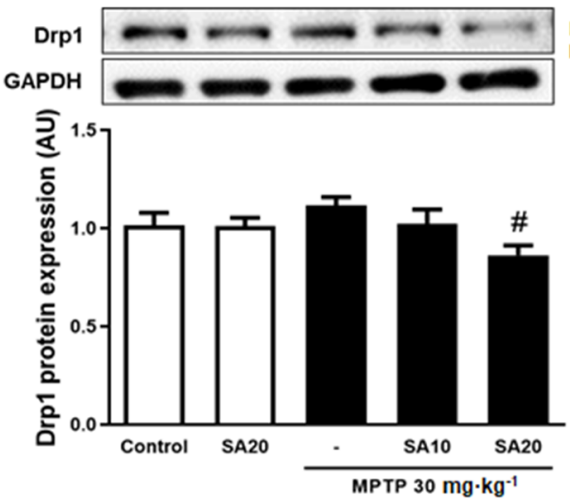

C
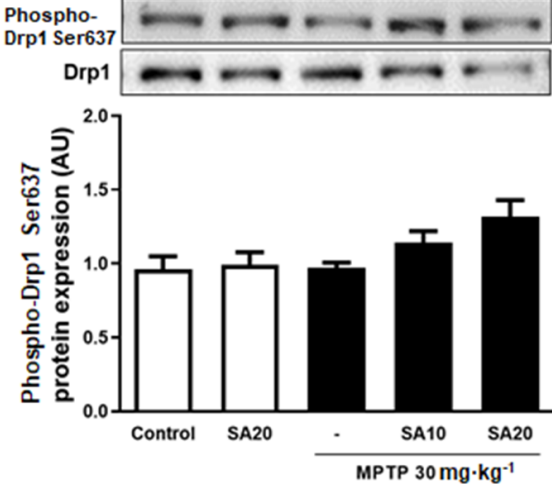

\section{B}

PhosphoDrp1 Ser616
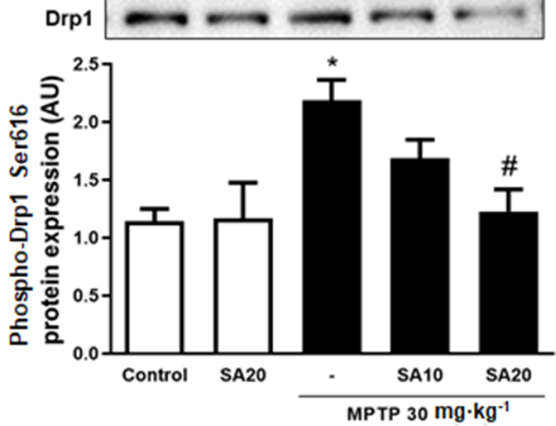

\section{D}

PhosphoDrp1 Ser616 Phospho-

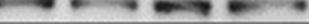
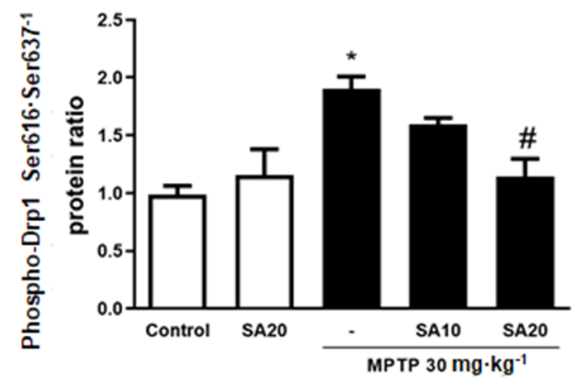
A

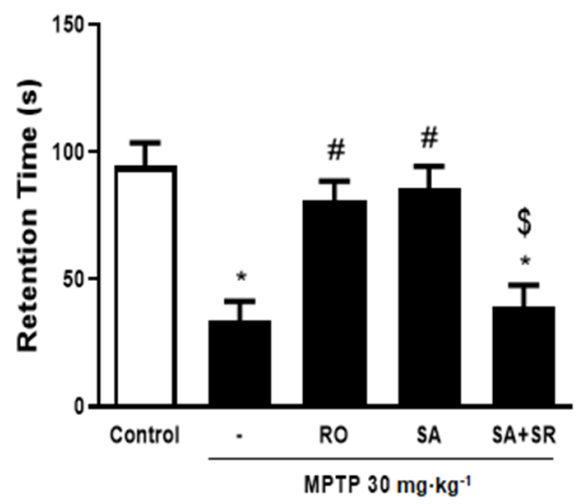

C
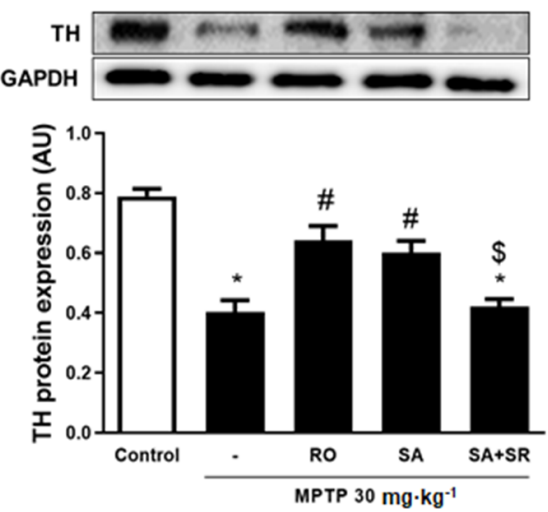

E

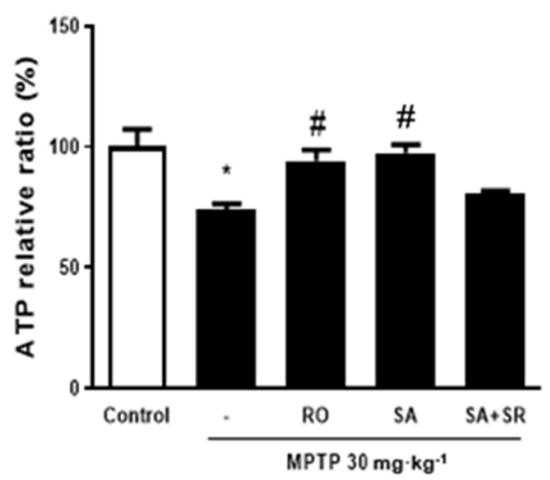

B

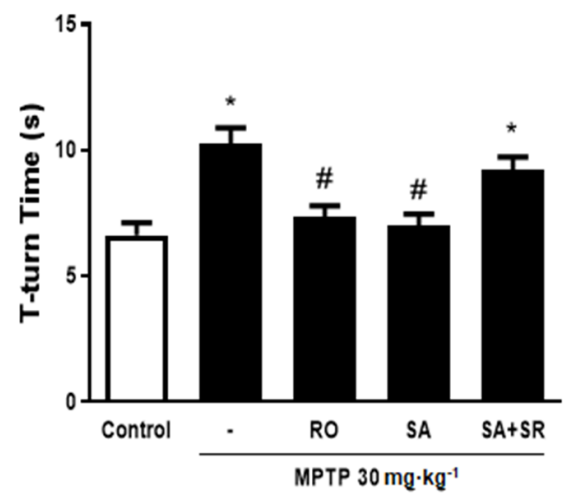

D
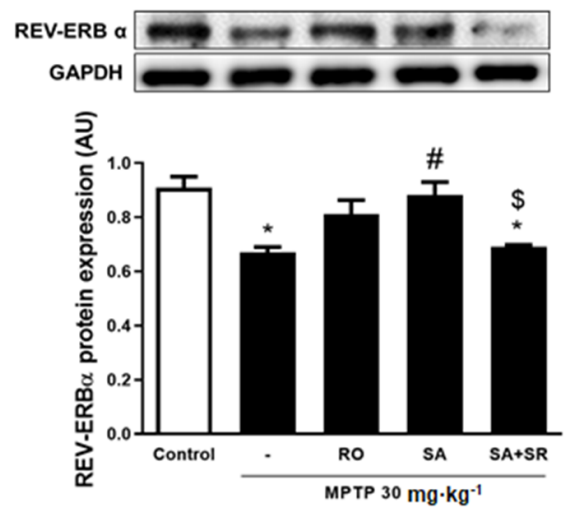

F

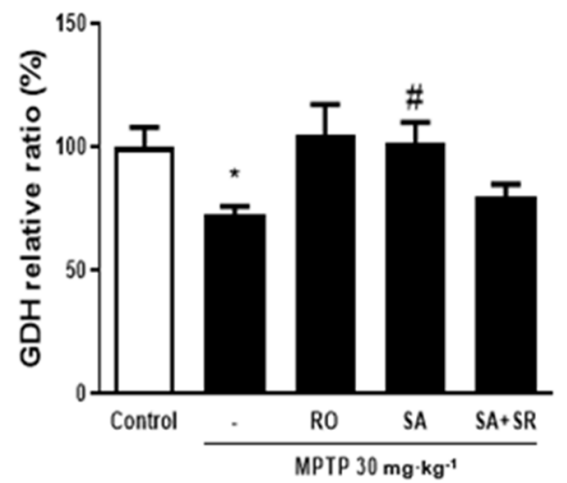


A

B

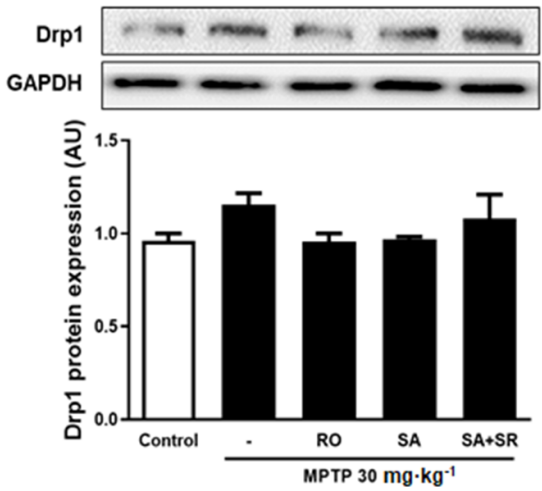

Phospho-

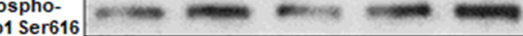

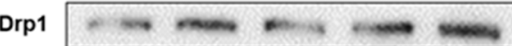

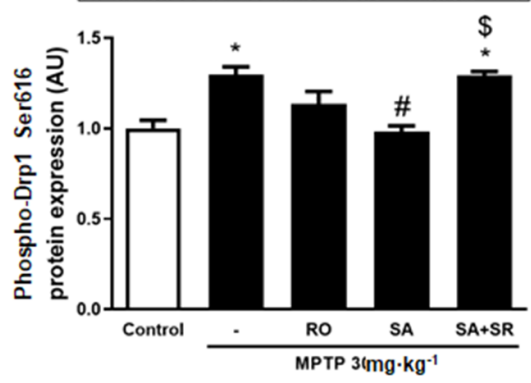

C

D

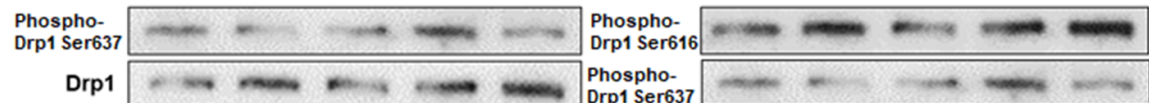
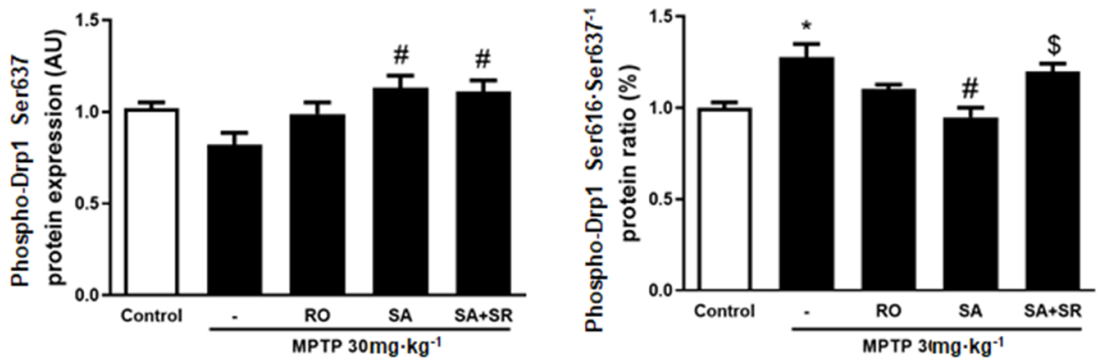